\title{
Effect of Chemical Reaction, Thermo-Diffusion, Hall Effects on MHD Convective Heat and Mass Transfer Flow of a Viscous Fluid in a Vertical Channel Bounded by Stretching and Stationary Walls
}

\author{
K.Chandrasekhar Raju ${ }^{1}$, Dr. B. Sreenivasa Reddy ${ }^{2 *}$ \\ ${ }^{I}$ Research scholar, Department of Mathematics, Dravidian University, Kuppam, A.P \\ ${ }^{2}$ Assistant Professor, Department of Applied Mathematics, Yogi Vemana University, Kadapa, A.P., India \\ *Corresponding Author: B. Sreenivasa Reddy, Assistant Professor, Department of Applied Mathematics, \\ Yogi Vemana University, Kadapa, A.P., India
}

\begin{abstract}
In this chapter, we investigate the effect of Chemical reaction, thermo-diffusion and Hall effects on hydromagnetic convective and mass transfer flow of an electrically conducting, viscous, incompressible fluid through a porous medium in a vertical channel bounded by stretching and stationary walls. The nonlinear governing equations have been solved by using Runge-Kutta shooting technique.
\end{abstract}

Keywords: Chemical Reaction, Heat and Mass transfer, Vertical Channel, Stretching sheet Thermo-diffusion

\section{INTRODUCTION}

MHD problems occur in several situations which include the prediction of space weather, damping of turbulent fluctuations in semiconductor melts in crystal growth, measurement of flow rates of beverages in food industry. MHD channel flows gained significant theoretical and practical importance owing to their applications in MHD generators, accelerators and blood flow measurements. Investigations for studies on MHD flow and heat transfer of non-Newtonian fluid flows generated by a stretching sheet find many applications in engineering and industry. For, example, in the extrusion of molten polymers, through a slit die for the manufacture of plastic sheets, the sheet is sometimes stretched. The properties of the final product in such processes mainly depend on the rate of cooling. If such a sheet in an electrically conducting Casson fluid under the influence of a magnetic field is drawn, the rate of cooling can be controlled, so that the end product can be obtained with the desired quality.

Crane [7] discussed the steady two dimensional incompressible boundary layer flow of a viscous fluid generated by an elastic flat sheet which moves in its plane with velocity varying linearly with distance from a fixed point due to the application of a uniform stress and obtained closed form solution. Mishra [18] discussed the study of MHD boundary layer flow of a viscous fluid over a stretching surface in the presence of a uniform transverse magnetic field with Hall currents. The study of mass transfer is significant in problems of convective heat transfer of atoms and molecules. Evaporation of water, separation of chemicals in distillation processes involves the applications in chemical and hydrometallurgical industries. Chemical reaction can be considered as homogeneous or heterogeneous processes. It depends on the reaction that takes place at the interface as a single phase volume reaction [19]. Several researchers [Kandaswamy et al [12], Hayat [10], Bhattacharya and Layek [4], Makinde[17], Ashraf[1], Asia[2], Raftari[23], Shrestha[27,28], Laurent[15], Qi et al[22]] studied the problems of mass transfer in the presence of chemical reaction.

Laminar flows through channels have applications in the fields of gas diffusion, ablation cooling, filtration, microfluid devices, surface sublimation, grain regression [as in the case of combustion in rocket motors] and the modelling of air circulation in the respiratory system [10]. Laminar air -flow systems have been used by the aerospace industry to control particular contamination. Furthermore, the laminar flow cabinet have been used in the maintenance of negative pressure and in the adjustment of 
the fans to exhaust more air. Therefore, the Navier-Stokes equations which are the governing equations for these problems, have attracted the interest of the researchers. Sutton and Barto[30] described an exact solution of Navier-Stokes equations for motion of an incompressible viscous fluid in a channel with different pressure gradients. Their solutions are helpful in verifying and validating computational models of complex on unsteady motions, to guide the design of fuel injectors and controlled experiments. Simulation of flow through micro-channels with design roughness was presented numerically by Rawool et al [24] A numerical investigation is made by Robinson [25] for the problem of steady laminar incompressible flow in a porous channel with uniform suction at both walls. Taylor et al [31] studied three dimensional flow by uniform suction through parallel porous walls. The investigations of Taylor [31] were further extended to a more general three dimensional stagnation point which can capture the phenomena in a single class of state by Hewitt et al [11]. Two dimensional viscous incompressible fluid flow between two porous walls with uniform suction was analysed by Cox [6]. Berman[3] proposed the two dimensional laminar steady flow through a porous channel which was driven by suction or injection .Similarity one/two dimensional laminar flow in a porous channel with wall suction or injection was examined analytically by Laurent et al[9]. The problem of fluid flow in a channel with porous walls was solved by Karode[13].Zheng et al[36] investigated asymptotic solutions for steady laminar flow of an incompressible viscous fluid along a channel with accelerating rigid porous walls. The exact solution sol for two dimensional steady laminar flow through a porous channel was generalized by Terril [32], Brady [5],Waston et al[34] and Cox[6] under varied conditions. Deng and Marinez [8] worked on two dimensional flow of a viscous fluid in a channel partially filled with porous medium with wall suction.

Wang [33] worked on viscous flow due to stretching sheet with slip and suction and proved a closed form unique solution for two dimensional flows. For axisymmetric stretching both existence and uniqueness were shown. Muhammad Asraf et al [20] investigated micropolar fluid flow in a channel with Shrinking walls. Haipour and Dehkordi [9] studied the transient behaviour of fluid flow and heat transfer in vertical channel partially filled with a porous medium including the effects of inertial term and viscous dissipation. Kasif Ali et al [14] have discussed numerical study of micropolar fluid flow and heat transfer in a channel with shrinking and stationary wall. Sarojamma et al [26] have studied MHD Casson fluid flow, heart and mass transfer in a vertical channel with stretching walls. Recently, Pudi Sreenivasa Rao [21] has discussed the effect of chemical reaction and dissipation on MHD convective heat and mass transfer flow in a vertical channel bounded by stretching and stationary walls in the presence of heat sources. Madhavi et al [16] have investigated heat and mass transfer flow of a rotating fluid in a vertical channel with stretching and stationary walls. Sukanya et al [29] have analysed Convective Heat and Mass Transfer Flow in a Rotating Vertical Channel Bounded by Stretching and Stationary Walls with Soret Effect and Thermal Radiation, Chemical Reaction in the Presence NonUniform Heat Sources the mixed convective heat and mass transfer flow in a vertical channel bounded at stretching walls.

In this chapter, we investigate the effect of Chemical reaction, thermo-diffusion and Hall effects on hydromagnetic convective and mass transfer flow of an electrically conducting, viscous, incompressible fluid through a porous medium in a vertical channel bounded by stretching and stationary walls. The nonlinear governing equations have been solved by using Runge-Kutta shooting technique. The velocity, temperature and concentration have been analysed for different variations of $\mathrm{G}, \mathrm{M}, \mathrm{m}, \mathrm{N}, \mathrm{Nr}, \mathrm{Sc}, \gamma, \mathrm{A} 1, \mathrm{~B} 1$, ,Sr and Pr The rate of Skin friction, rate of heat and mass transfer on the walls are evaluated numerically for different variations.

\section{FORMULATION OF THE PROBLEM}

We consider the steady two dimensional hydro-magnetic laminar convective heat and mass transfer flow of a viscous electrically conducting fluid through a porous medium in a vertical channel bounded by a stretching sheet on the left a stationary plate on the right. We choose a rectangular coordinate system $\mathrm{O}(\mathrm{x}, \mathrm{y} . \mathrm{z})$ with the walls at $\mathrm{y}= \pm \mathrm{L}$. The flow is subjected to a string magnetic field with a constant intensity Bo along the positive y-direction. Assuming magnetic Reynolds number Rm to be small we neglect the induced magnetic field in comparison to the applied magnetic field. It is used to compare the transport of magnetic lines of the force in a conducting fluid with the leakage of such lines from the fluid. 


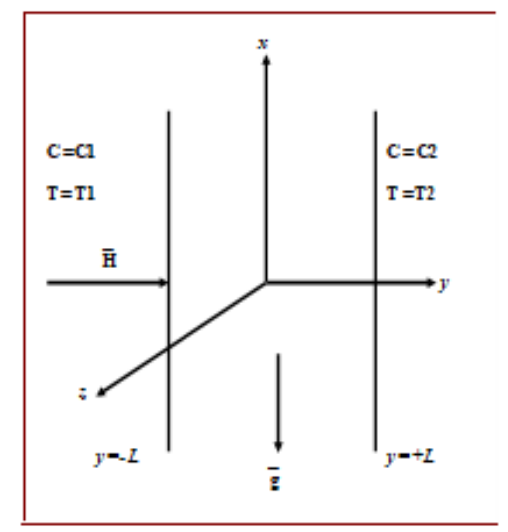

Fig1. Configuration of the problem

The magnetic field $\nabla \cdot \bar{H}=0$, where $\bar{H}=\left(H_{x}, H_{y}, H_{z}\right)$ gives $H_{y}=H_{0}($ cons $\tan t)$ everywhere in the flow field, which gives $\bar{H}=\left(0, H_{0}, 0\right)$.If $\bar{J}=\left(J_{x}, J_{y}, J_{z}\right)$ is the current density vector, the equation of conservation of electric charge $\nabla . \bar{J}=0_{\text {gives }}{ }^{J_{y}}=$ constant . This constant is zero, that is, ${ }^{J^{y}}=0$ everywhere in the flow field. Since the walls are electrically non-conducting. The generalized Ohm's law, in the absence of electric field (22), is of the form

$\bar{J}+\frac{\omega_{e} \tau_{e}}{H_{o}}(\bar{J} x \bar{H})=\sigma\left(\mu_{e} \bar{q} x \bar{H}+\frac{1}{e n_{e}} \nabla p_{e}\right)$

Where $\bar{q}, \sigma, \mu_{e}, \omega_{e}, \tau_{e}$ e, ne and pe are the velocity, the electrical conductivity, the magnetic permeability, the cyclotron frequency, the electron collision time, the electric charge, the number density of the electron and the electron pressure respectively. Under the usual assumptions, the electron pressure (for a weakly ionized gas), the thermoelectric pressure and ion slip are negligible, so we have from the Ohm's law

$$
\begin{aligned}
& j_{x}+\omega_{e} \tau_{e} j_{z}=\sigma \mu_{e} H_{o} w \\
& j_{z}-\omega_{e} \tau_{e} j_{x}=-\sigma \mu_{e} H_{o} u
\end{aligned}
$$

From which we obtain that

$$
\begin{aligned}
& j_{x}=\frac{\sigma \mu_{e} H_{o}(m u+w)}{1+m^{2}} \\
& j_{z}=\frac{\sigma \mu_{e} H_{o}(m w-u)}{1+m^{2}} \\
& \bar{q}=(u(x, y), v(x, y), w(x, y)), T=T(x, y), C=C(x, y)
\end{aligned}
$$

Taking the viscous dissipation and joule heating effects into consideration, the governing equations of the flow, heat and mass transfer for the problem are

$$
\begin{aligned}
& \frac{\partial u}{\partial x}+\frac{\partial v}{\partial y}=0 \\
& \rho\left(u \frac{\partial u}{\partial x}+v \frac{\partial u}{\partial y}\right)=-\frac{\partial p}{\partial x}+\mu\left(\frac{\partial^{2} u}{\partial x^{2}}+\frac{\partial^{2} u}{\partial y^{2}}\right)-\frac{\sigma \mu_{e}^{2} H_{o}^{2}}{1+m^{2}}(u+m w)-\rho g \\
& \rho\left(u \frac{\partial v}{\partial x}+v \frac{\partial v}{\partial y}\right)=-\frac{\partial p}{\partial y}+\mu\left(\frac{\partial^{2} v}{\partial x^{2}}+\frac{\partial^{2} v}{\partial y^{2}}\right)
\end{aligned}
$$




$$
\begin{aligned}
& \rho\left(u \frac{\partial w}{\partial x}+v \frac{\partial w}{\partial y}\right)=\mu\left(\frac{\partial^{2} w}{\partial x^{2}}+\frac{\partial^{2} w}{\partial y^{2}}\right)+\frac{\sigma \mu_{e}^{2} H_{o}^{2}}{1+m^{2}}(m u-w) \\
& \rho C_{p}\left(u \frac{\partial T}{\partial x}+v \frac{\partial T}{\partial y}\right)=k_{f}\left(\frac{\partial^{2} T}{\partial x^{2}}+\frac{\partial^{2} T}{\partial y^{2}}\right)+q^{\prime \prime \prime}-\frac{\partial\left(q_{R}\right)}{\partial y} \\
& +2 \mu\left(\left(\frac{\partial u}{\partial y}\right)^{2}+\left(\frac{\partial w}{\partial y}\right)^{2}\right)+\sigma \mu_{e}^{2} H_{o}^{2}\left(u^{2}+w^{2}\right) \\
& \left(u \frac{\partial C}{\partial x}+v \frac{\partial C}{\partial y}\right)=D_{b}\left(\frac{\partial^{2} C}{\partial x^{2}}+\frac{\partial^{2} C}{\partial y^{2}}\right)-k_{c}^{\prime}\left(C-C_{o}\right)+\frac{D_{T} K_{T}}{T_{m}}\left(\frac{\partial^{2} T}{\partial x^{2}}+\frac{\partial^{2} T}{\partial y^{2}}\right) \\
& \rho-\rho_{o}=-\beta\left(T-T_{o}\right)-\beta^{*}\left(C-C_{o}\right)
\end{aligned}
$$

Where(u,v.0) are the velocity components along $\mathrm{x}, \mathrm{y}$ directions respectively, T, C are the dimensional temperature and concentration respectively, $\rho$ is the density, $p$ is the pressure, $\sigma$ is the electrical conductivity, $\mu_{e}$ is the magnetic permeability of the medium, $\mu$ is the dynamic viscosity, $\mathrm{g}$ is the gravity, is the strength of the heat source, $\mathrm{Db}$ is the molecular diffusivity, $\mathrm{D}_{\mathrm{T}}$ is the mass diffusivity, $\mathrm{K}_{\mathrm{T}}$ is the thermal diffusion ratio, $\beta$ is the coefficient of thermal expansion, $\beta^{*}$ is the coefficient of volume expansion, $\mathrm{Tm}$ is the mean fluid temperature, $m)=\omega_{e} \tau_{e} \quad$ is the Hall parameter, $\omega_{e}=e \frac{B_{o}}{m_{e}}$ is the electron frequency

The coefficient $q^{\prime \prime \prime}$ is the rate of internal heat generation $(>0)$ or absorption $(<0)$. The internal heat generation /absorption $q^{\prime \prime \prime}$ is modelled as

$$
q^{\prime \prime \prime}=\left(\frac{k u_{s}}{x v}\right)\left[A 1\left(T_{1}-T_{1}\right) f^{\prime}(\eta)+B 1\left(T-T_{2}\right)\right]
$$

Where A1 and B1 are coefficients of space dependent and temperature dependent internal heat generation or absorption respectively. It is noted that the case $\mathrm{A} 1>0$ and $\mathrm{B} 1>0$, corresponds to internal heat generation and that $\mathrm{A} 1<0$ and $\mathrm{B} 1<0$, the case corresponds to internal heat absorption case.

The boundary conditions for the velocity, temperature and concentration are

$$
\begin{aligned}
& u(x,-L)=u s=b x, u(x,+L)=0, v(x, \pm L)=0, \\
& T(x,-L)=T_{1}, T(x,+L)=T_{2} \\
& C(x,-L)=C_{1}, C(x,+L)=C_{2}
\end{aligned}
$$

Where $\mathrm{b}>0$ is the stretching rate of the channel wall, $\mathrm{T} 1, \mathrm{~T} 2$ (with $(\mathrm{T} 1>\mathrm{T} 2)$ are the fixed temperature of the left and right walls respectively, $\mathrm{C} 1, \mathrm{C} 2$ (with $\mathrm{C} 1>\mathrm{C} 2$ ) or the fixed concentrations of the channel walls respectively. We introduce the following Similarity variables as:

$$
\begin{aligned}
& \eta=\frac{y}{L} ; u=b x f^{\prime}(\eta) ; v=-b L f(\eta), w=b x g_{0}(\eta) \\
& \theta(\eta)=\frac{T-T_{2}}{T_{1}-T_{2}}, \phi(\eta)=\frac{C-C_{2}}{C_{1}-C_{2}}
\end{aligned}
$$

The above velocity field is compatible with continuity equation (2) and therefore, represents the possible fluid motion.

Eliminating the pressure between the equations (3) and (4) and using (7) \& (9) the momentum equation reduces to

$$
\begin{gathered}
f^{i v}+\operatorname{Re}_{x}\left(f^{\prime \prime \prime} f-f^{\prime} f^{\prime \prime}\right)-\frac{M^{2}}{1+m^{2}}\left(f^{\prime \prime}+m g_{0}{ }^{\prime}\right) \\
+G r\left(\theta^{\prime}+N \phi^{\prime}\right)=0
\end{gathered}
$$


$g_{0}^{i i}+\operatorname{Re}_{x}\left(f_{0} g^{\prime}-f^{\prime} g_{0}\right)+\frac{M^{2}}{1+m^{2}}\left(m f^{\prime}-g_{0}\right)=0$

where as the equations (6) \& (7) in view of equation (9) are

$$
\begin{aligned}
(1+4 R d / 3) \theta^{\prime \prime}+\operatorname{Pr} \operatorname{Re} x\left(f \theta^{\prime}\right) & +\left(A 1 f^{\prime}+B 1 \theta\right)+\operatorname{Pr} E c\left(\left(f^{\prime \prime}\right)^{2}+\left(g_{0}\right)^{2}\right)+ \\
& +\frac{M^{2} E c}{1+m^{2}}\left(\left(f^{\prime}\right)^{2}+g_{0}{ }^{2}\right)=0
\end{aligned}
$$

$\phi^{\prime \prime}+S c \operatorname{Re} x\left(f \phi^{\prime}\right)-\gamma \phi+S c S o \theta^{\prime \prime}=0$

Where $G r=\frac{\beta g\left(T_{1}-T_{2}\right) L}{b x}$, is the Grashof number, $N=\frac{\beta^{*}\left(C_{1}-C_{2}\right)}{\beta\left(T_{1}-T_{2}\right)}$, is the buoyancy ratio, $M^{2}=\frac{\sigma \mu_{e}^{2} H_{o}^{2} L^{2}}{v x}$, magnetic parameter, $D^{-1}=\frac{L^{2}}{k}$, Inverse Darcy parameter,

$\operatorname{Pr}=\frac{\mu C_{p}}{k_{f}}$ is Prandtl number, $\operatorname{Re} x=\frac{b L^{2}}{\mu}$, is the local Reynolds number,

$S c=\frac{v}{D_{m}}$, is the Schmidt number, $S o=\frac{D_{T} K_{T}\left(T_{1}-T_{2}\right)}{T_{m}\left(C_{1}-C_{2}\right)}$, is the Soret parameter

$\gamma=\frac{k_{c} L^{2}}{D_{m}}$ is the chemical reaction parameter, $E c=\frac{b^{2} x^{2}}{C_{p} \Delta T}$ is the Eckert number

Boundary conditions (8), in view of equation (10) in dimensional form reduces to

$f( \pm 1)=0, f^{\prime}(-1)=1, f^{\prime}(+1)=0, \mathrm{~g}_{0}(-1)=0, \mathrm{~g}_{0}(+1)=0$

$\theta(-1)=1, \theta(+1)=0, \phi(-1)=1, \phi(+1)=0$,

\section{Method of Solution}

A usual approach is to write the nonlinear ODE in form of a first order initial value problem as follows:

$$
\begin{aligned}
& f=f_{1}, f^{\prime}=f_{2} f^{\prime \prime}=f_{3}, f^{\prime \prime \prime}=f_{4}, g=f_{5}, g^{\prime}=f_{6} \\
& \theta=f_{7}, \theta^{\prime}=f_{8}, \phi=f_{9}, \phi^{\prime}=f_{10} \\
& f^{i v}=f_{4}^{\prime}=-\operatorname{Re} x\left(f_{4} f_{1}-f_{2} f_{3}\right)-\frac{M^{2}}{1+m^{2}}\left(m f_{6}+f_{3}\right)+ \\
& +G r\left(f_{6}+N f_{8}\right) \\
& g^{i i}=f_{6}^{\prime}=-\operatorname{Re} x\left(f_{6} f_{1}-f_{2} f_{5}\right)-\frac{M^{2}}{1+m^{2}}\left(m f_{2}-g\right) \\
& \theta^{\prime \prime}=f_{6}^{\prime}=\left[-\operatorname{Pr} \operatorname{Re} x\left(f_{1} f_{6}\right)+\left(A 1 f_{2}+B 1 f_{7}\right)-\operatorname{Pr} E c\left(f_{3}^{2}+f_{6}^{2}\right)-\right. \\
& -M^{2} E c\left(f_{2}^{2}+f_{5}^{2}\right) \\
& \phi^{\prime \prime}=f_{8}^{\prime}=\left[-S c \operatorname{Re} x\left(f_{1} f_{10}\right)+\gamma f_{9}-S c S o\left(-\operatorname{Pr} \operatorname{Re}_{x}\left(f_{1} f_{8}\right)-\left(A 1 f_{2}+B 1 f_{7}\right)-\right.\right. \\
& \left.-\frac{M^{2} E c}{1+m^{2}}\left(f_{2}^{2}+f_{5}^{2}\right)-\operatorname{Pr} E c\left(f_{3}^{2}+f_{5}^{2}\right)\right] /(1+S c S r)
\end{aligned}
$$

The corresponding boundary conditions are

$f_{1}( \pm 1)=0, f_{2}(-1)=1, f_{2}(+1)=0, f_{5}(-1)=1, f_{5}(+1)=0$, 
$f_{6}(-1)=1, f_{6}(+1)=0, f_{7}(-1)=1$,

$f_{7}(+1)=0, f_{8}(-1)=1, f_{8}(+1)=0$,

Here $f_{3}(-1), f_{4}(-1), f_{6}(-1), f_{8}(-1), f_{10}(-1)$ are the unknown initial condition, Therefore, a shooting methodology is incorporated to solve the above system, which may be a combination of the RungeKutta method(to solve first order ODE) and a five dimensional zero finding algorithm(to find the missing coordinates).It is note that the missing initial conditions are coupled so that the solution satisfies the boundary conditions $f(+1)=0, f^{\prime}(+1)=0, g(+1)=0, \theta(+1)=0, \phi(+1)=0$ of the original boundary value problem.

\section{COMPARISON}

In absence of hall effect $(\mathrm{m}=0)$, heat sources $(\mathrm{A} 1=0, \mathrm{~B} 1=0)$ the results are good aggrement with Sreenivasa Rao [21].

Table1.

\begin{tabular}{|c|c|c|c|c|c|c|c|c|c|c|}
\hline & & & & & Sree & Rao Re & [21] & & esent Resul & \\
\hline & & & & & & & & & & \\
\hline Rd & $\mathrm{Sr}$ & Ec & $\gamma$ & Pr & & & & & & \\
\hline 0.5 & 0.5 & 0.01 & 0.5 & 0.71 & $\begin{array}{c}- \\
0.0479754\end{array}$ & 2.13075 & 0.585837 & $\begin{array}{c}- \\
0.0479654\end{array}$ & 2.13069 & 0.585834 \\
\hline 1.5 & 0.5 & 0.01 & 0.5 & 71 & -0.056628 & 1.29034 & 0.511647 & -0.056619 & 1.29039 & 0.511644 \\
\hline 3.5 & 0.5 & 0. & 0.5 & 0. & $\begin{array}{c}- \\
0.0665265\end{array}$ & 0.410303 & 0.373097 & $\begin{array}{c}- \\
0.0665245\end{array}$ & 0.410329 & .373096 \\
\hline 0.5 & 1.0 & 0.01 & 0.5 & 0.71 & -0.042911 & 0.131989 & 0.903708 & -0.042909 & 0.131979 & 0.903706 \\
\hline 0.5 & 1.5 & 0.01 & 0.5 & 0.71 & $\begin{array}{c}- \\
0.0510722 \\
\end{array}$ & 0.123694 & 1.77443 & $\begin{array}{c}- \\
0.0510769 \\
\end{array}$ & 0.123666 & 1.77439 \\
\hline 0.5 & 0.5 & 0 . & 0.5 & 0.71 & $\begin{array}{c}- \\
0.0406445\end{array}$ & 63 & 12 & $\begin{array}{c}- \\
0.0406439\end{array}$ & 54 & 105 \\
\hline 0.5 & 0.5 & 0.05 & 0.5 & 0.71 & $\begin{array}{c}- \\
0.0411362 \\
\end{array}$ & 0.143238 & 0.629183 & $\begin{array}{c}- \\
0.0411359 \\
\end{array}$ & 0.143224 & 0.629169 \\
\hline 0.5 & 0.5 & 0.01 & 1.5 & 0.71 & $\begin{array}{c}- \\
0.0361883\end{array}$ & 0.139816 & 0.26852 & $\begin{array}{c}- \\
0.0361879\end{array}$ & 0.139812 & 0.26849 \\
\hline 0.5 & 0.5 & 0.01 & $\begin{array}{c}- \\
0.5\end{array}$ & 0.71 & 0.0655513 & 0.184642 & 13 & 0.0655508 & 0.184639 & 05 \\
\hline 0.5 & 0.5 & 0.01 & - & 0.71 & .112906 & 0.144466 & 5.48746 & 12904 & 0.144459 & 5.48744 \\
\hline 0.5 & 0.5 & 0.01 & 0.5 & 1.71 & $\begin{array}{c}- \\
0.0219619\end{array}$ & 0.0251027 & 0.568978 & $\begin{array}{c}-\overline{-} \\
0.0219604\end{array}$ & 0.0251025 & 0.568966 \\
\hline 0.5 & 0.5 & 0.01 & 0.5 & 3.71 & 0.0156345 & 0.0050031 & 0.467665 & $\begin{array}{c}- \\
0.0156339\end{array}$ & 0.0050029 & 0.467668 \\
\hline 0.5 & 0.5 & 0.01 & 0.5 & 7.0 & $\begin{array}{c}- \\
0.0117748\end{array}$ & 0.00 & 0.3 & $\begin{array}{c}\dot{-} \\
0.0117735\end{array}$ & 576 & 729 \\
\hline
\end{tabular}

\section{Discussion of Numerical Results}

In this analysis we investigate the influence of Hall effects, thermo-diffusion and non-uniform heat sources, chemical reaction on MHD convective heat and mass transfer flow of a viscous, dissipative fluid in a vertical channel bounded by a stretching sheet and stationary plates. The non-linear governing equations have been solved by employing Fourth order Runge-Kutta -Shooting technique.

The primary velocity, secondary velocity, temperature and concentration have been analysed for different variations of $\mathrm{m}, \mathrm{Rd}, \mathrm{A} 1, \mathrm{~B} 1, \mathrm{Ec}$, So and $\gamma$.

The primary velocity $\left(f^{\prime}(\eta)\right.$, Secondary velocity $(g(\eta))$, temperature $(\theta)$ and concentration $(\phi)$ are shown in Figs.2a-2d demonstrate $f^{\prime}, g, \theta$ and $\phi$ with Hall parameter $(m)$. As mentioned above, the Lorentz 
force has retarding effect on the primary velocity. This retarding is reduced with increase in the Hall parameter and hence the primary velocity is enhanced and consequently the boundary layer becomes thicker. The secondary velocity increases as the Hall parameter $m$ increases. The effect of Hall parameter on temperature and concentration is observed to be opposite to that of magnetic field.

Figs.3a-3d shows the variation of $\mathrm{f}^{\prime}, \mathrm{g}, \theta$ and $\phi$ with radiation parameter(Rd).It can seen from the profiles that higher the radiative heat flux larger the primary and secondary velocities.The effect of thermal radiation is enhance the temperature and reduces the concentration in the flow region.

Figs.4a-4d,illustrates $\mathrm{f}^{\prime}, \mathrm{g}, \theta, \phi$ with variation in space dependent Heat source parameter $(\mathrm{A} 1>0)$. The presence of the heat sources generates energy in the momentum boundary layer, thermal boundary layer and absorbs the energy in solutal boundary layer and as a consequence the primary velocity, temperature enhances in the boundary layers and reduces the secondary velocity and concentration. In the case of heat absorption $(\mathrm{A} 1<0)$ the velocity components, temperature reduces and the concentration rises with decreasing values of $A 1<0$, owing to the absorption of energy in the boundary layer.

Figs.5a-5d,illustrates $\mathrm{f}^{\prime}, \mathrm{g}, \theta, \phi$ with variation in temperature dependent heat source parameter $(\mathrm{B} 1>0)$. As in the case of space dependent heat source, the velocity components, temperature and concentration increase due to the release of energy for $\mathrm{B} 1>0$ while the velocity components, temperature drop and the concentration rise for decreasing values of $\mathrm{B} 1<0$ and owing to the absorption of energy. The presence of the temperature dependent heat sources generates energy in the momentum boundary layer, thermal boundary layer and solutal boundary layer and as a consequence the velocity components, temperature and concentration enhances in the boundary layers. In the case of heat absorption $(\mathrm{B} 1<0)$ the velocity components, temperature depreciate and concentration rises with decreasing values of $\mathrm{B} 1<0$, owing to the absorption of energy in the momentum and thermal boundary layers and generates energy in the solutal boundary layer.

The effect of dissipation (Ec) on $\mathrm{f}^{\prime}, \mathrm{g},, \theta$ and $\mathrm{C}$ is shown in figs.6a-6d. It can be found that higher the dissipative energy larger the magnitude of the velocity components, temperature and concentration in the flow region. This is due to the fact that heat energy is reserved due to frictional heating.

Figs.7a-7d,8a-8d show the variation of primary velocity, secondary velocity, temperature and concentration with chemical reaction parameter $(\gamma)$.It can seen from the profiles that the primary velocity and the temperature (figs.7a,7c) and the secondary velocity, concentration reduce in the degenerating chemical reaction case (figs.7b,7d) and in the generating chemical reaction case the velocity components and the concentration enhances(figs.8a,8c) and the temperature reduces in the entire flow region(figs.8b,8d).

Figs.9a-9d represent $\mathrm{f}^{\prime}, \mathrm{g},, \theta$ and $\mathrm{C}$ with Soret parameter So. It can be seen from the profiles that higher the thermo-diffusion effects larger the magnitude of velocity components, concentration and smaller the temperature in the flow region. This can be attributed to the fact that increasing values of So increases the thickness of the momentum boundary layer and solutal boundary layer and reduces the thermal boundary layer.

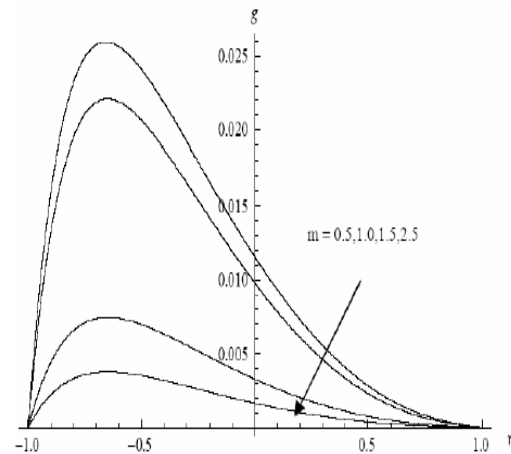

Fig. $2 b$ : Variation of $g_{0}$ with $m$

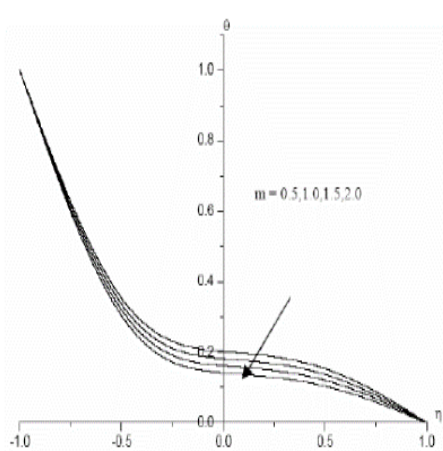

Fig.2c : Variation of $\theta$ with $m$

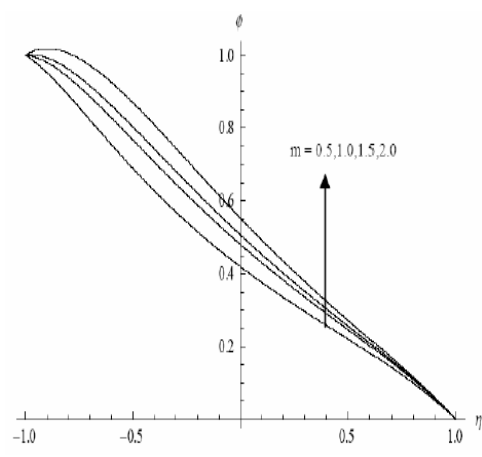

Fig.2d: Variation of $\phi$ with $m$

$\mathrm{A}_{1}=0.1, \mathrm{~B}_{1}=0.1, \gamma=0.5, \mathrm{Rd}=0.5, \mathrm{Ec}=0.01, \mathrm{~S}_{0}=0.5 \quad \mathrm{~A}_{1}=0.1, \mathrm{~B}_{1}=0.1, \gamma=0.5, \mathrm{Rd}=0.5, \mathrm{Ec}=0.01, \mathrm{~S}_{0}=0.5 \quad \mathrm{~A}_{1}=0.1, \mathrm{~B}_{1}=0.1, \gamma=0.5, \mathrm{Rd}=0.5, \mathrm{Ec}=0.01, \mathrm{~S}_{0}=0.5$ 


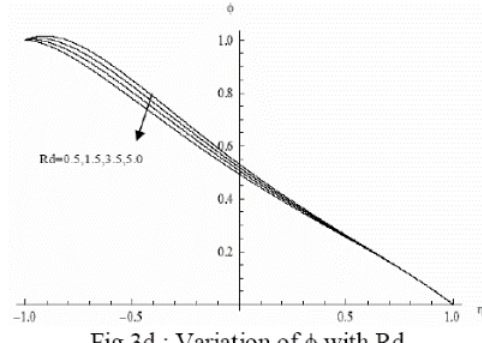

Fig.3d : Variation of $\phi$ with Rd $\mathrm{m}=0.5, \mathrm{~B}_{1}=0.1, \gamma=0.5, \mathrm{Ec}=0.01, \mathrm{So}=0.5, \mathrm{~A}=0.1$

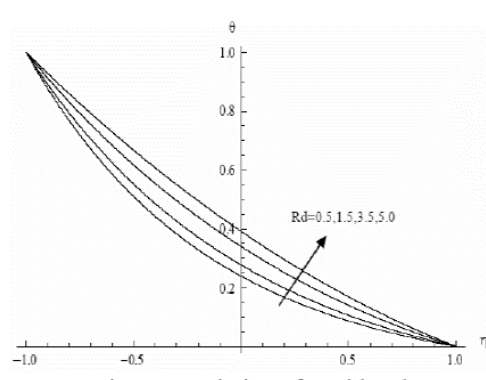

Fig.3c : Variation of $\theta$ with $R d$ $\mathrm{m}=0.5, \mathrm{~B}_{1}=0.1, \gamma=0.5, \mathrm{Ec}=0.01, \mathrm{So}=0.5, \mathrm{~A}=0.1$

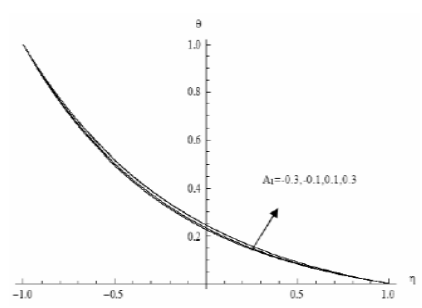

Fig. $4 \mathrm{c}$ : Variation of $\theta$ with $\mathrm{A}_{1}$ $\mathrm{m}=0.5, \mathrm{~B}_{1}=0.1, \gamma=0.5, \mathrm{Ec}=0.01, \mathrm{So}_{0}=0.5, \mathrm{Rd}=0.5$

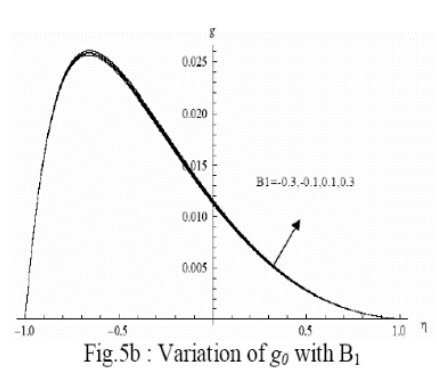
$\mathrm{m}=0.5, \mathrm{~A}_{1}=0.1, \gamma=0.5, \mathrm{Ec}=0.01, \mathrm{So}_{0}=0.5, \mathrm{Rd}=0.5$

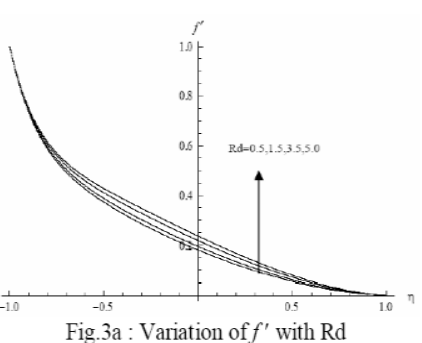

$\mathrm{m}=0.5, \mathrm{~B}_{1}=0.1, \gamma=0.5, \mathrm{Ec}=0.01, \mathrm{So}=0.5, \mathrm{~A}=0.1$

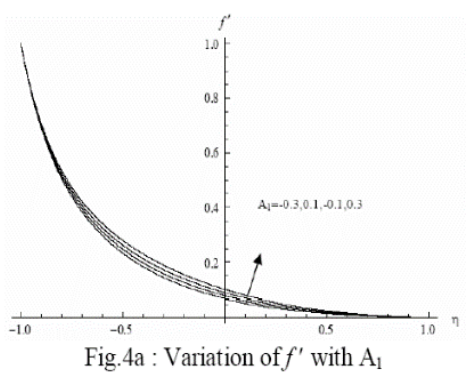

$\mathrm{m}=0.5, \mathrm{~B}_{1}=0.1, \gamma=0.5, \mathrm{Ec}=0.01, \mathrm{So}=0.5, \mathrm{Rd}=0.5$

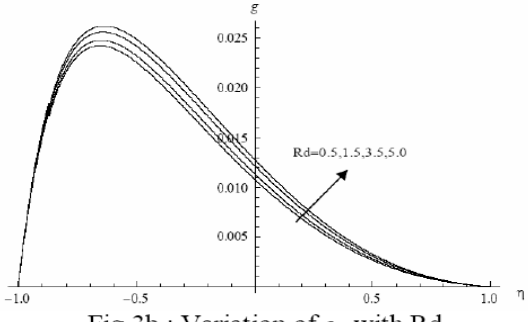

Fig. $3 b$ : Variation of $g_{0}$ with $\mathrm{Rd}$ $\mathrm{m}=0.5, \mathrm{~B}_{1}=0.1, \gamma=0.5, \mathrm{Ec}=0.01, \mathrm{So}=0.5, \mathrm{~A}=0.1$

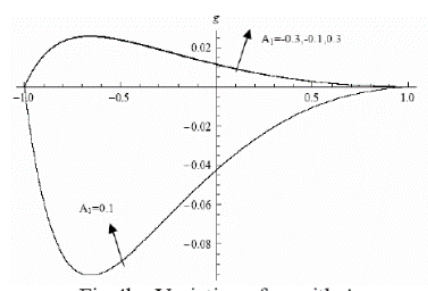

Fig. $4 \mathrm{~b}$ : Variation of $g_{0}$ with $\mathrm{A}_{1}$ $\mathrm{m}=0.5, \mathrm{~B}_{1}=0.1, \gamma=0.5, \mathrm{Ec}=0.01, \mathrm{So}=0.5, \mathrm{Rd}=0.5$

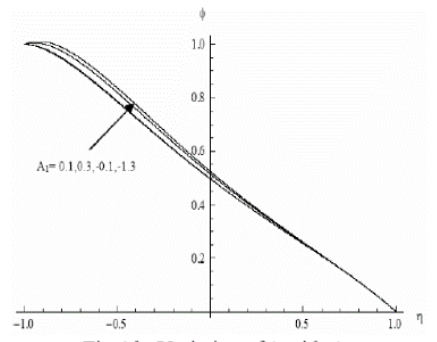

Fig.4d : Variation of $\phi$ with $A_{1}$ $\mathrm{m}=0.5, \mathrm{~B}_{1}=0.1, \gamma=0.5, \mathrm{Ec}=0.01, \mathrm{So}=0.5, \mathrm{Rd}=0.5 \quad \mathrm{~m}=0.5, \mathrm{~A}_{1}=0.1, \gamma=0.5, \mathrm{Ec}=0.01, \mathrm{So}_{0}=0.5, \mathrm{Rd}=0.5$

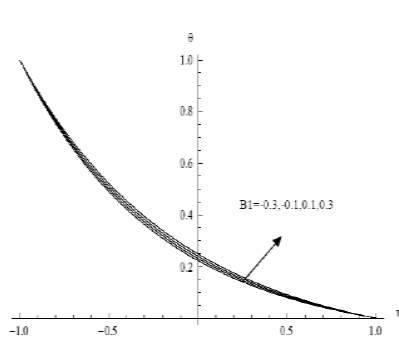

Fig. $5 \mathrm{c}$ : Variation of $\theta$ with $\mathrm{B}_{1}$

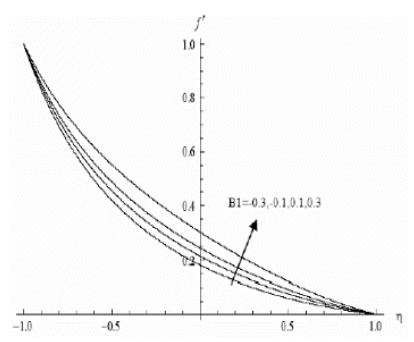

Fig.5a : Variation of $f^{\prime}$ with $\mathrm{B}$

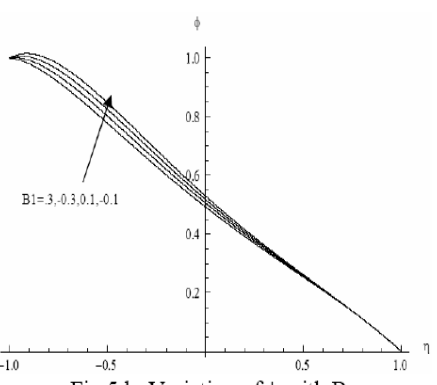

Fig. $5 d$ : Variation of $\phi$ with $B_{1}$ $\mathrm{m}=0.5, \mathrm{~A}_{1}=0.1, \gamma=0.5, \mathrm{Ec}=0.01, \mathrm{So}_{0}=0.5, \mathrm{Rd}=0.5 \mathrm{~m}=0.5, \mathrm{~A}_{1}=0.1, \gamma=0.5, \mathrm{Ec}=0.01, \mathrm{So}_{0}=0.5, \mathrm{Rd}=0.5$
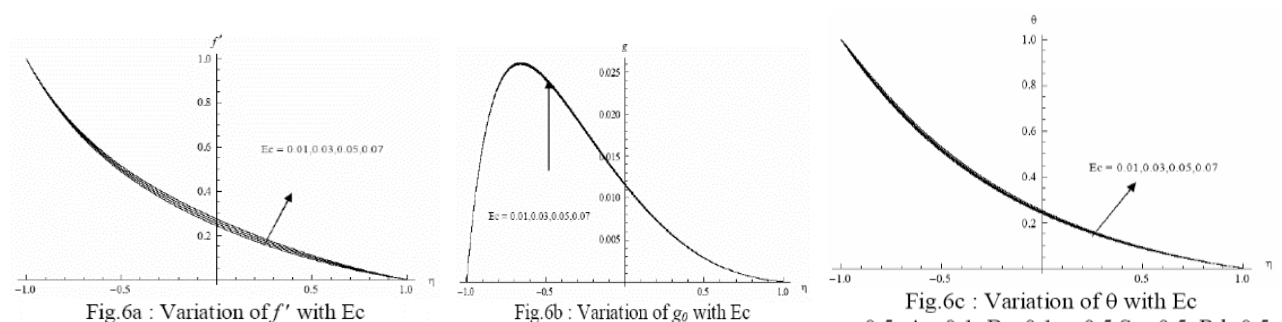

$\mathrm{m}=0.5, \mathrm{~A}_{1}=0.1, \mathrm{~B}_{1}=0.1, \gamma=0.5, \mathrm{So}=0.5, \mathrm{Rd}=0.5$

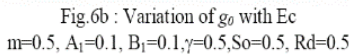

$\mathrm{m}=0.5, \mathrm{~A}_{1}=0.1, \mathrm{~B}_{1}=0.1, \gamma=0.5, \mathrm{So}=0.5, \mathrm{Rd}=0.5$
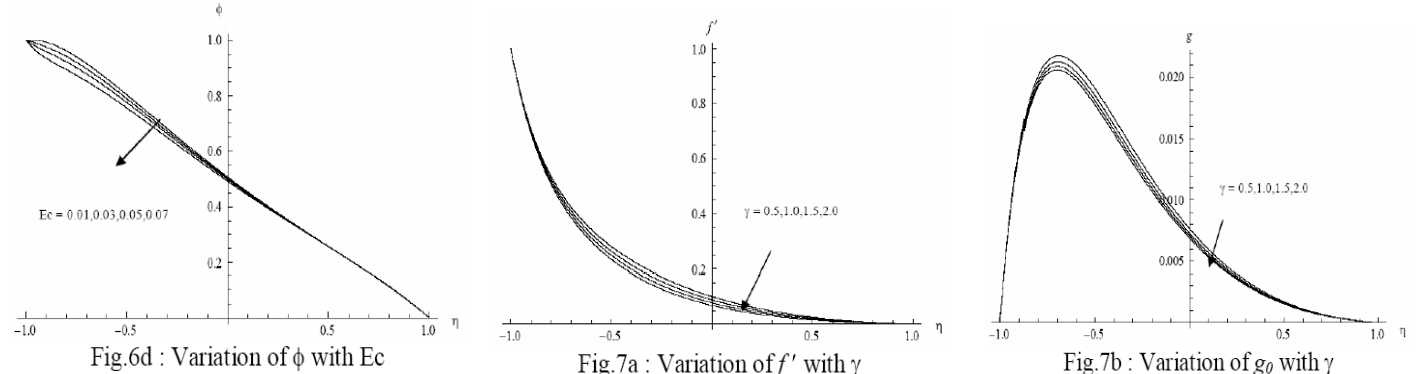

$\mathrm{m}=0.5, \mathrm{~A}_{1}=0.1, \mathrm{~B}_{1}=0.1, \gamma=0.5, \mathrm{So}=0.5, \mathrm{Rd}=0.5 \quad \mathrm{~m}=0.5, \mathrm{~A}_{1}=0.1, \mathrm{~B}_{1}=0.1, \mathrm{Ec}=0.01 . \mathrm{So}=0.5, \mathrm{Rd}=0.5 \mathrm{~m}=0.5, \mathrm{~A}_{1}=0.1, \mathrm{~B}_{1}=0.1, \mathrm{Ec}=0.01, \mathrm{So}=0.5, \mathrm{Rd}=0.5$ 

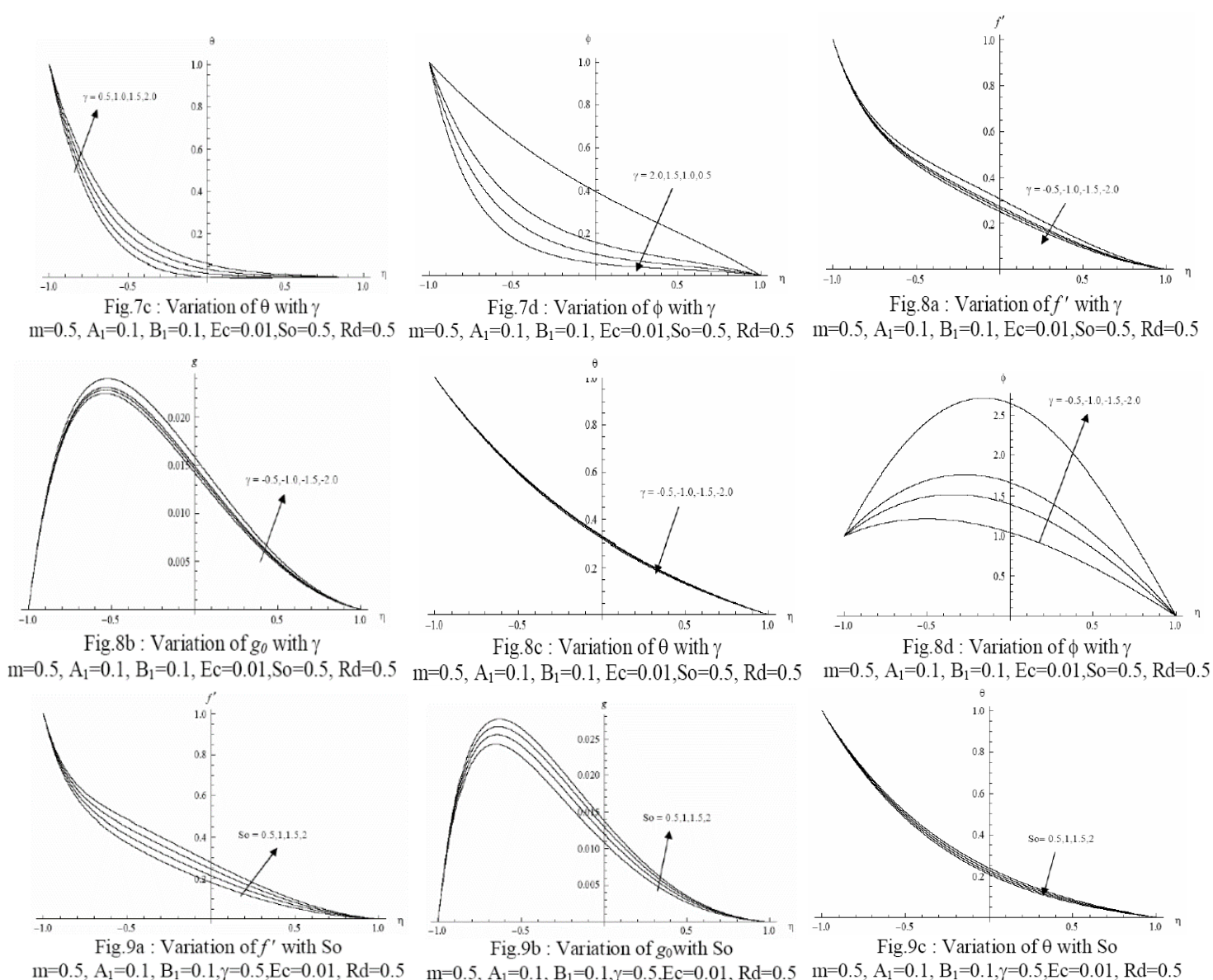

$\mathrm{m}=0.5, \mathrm{~A}_{1}=0.1, \mathrm{~B}_{1}=0.1, \gamma=0.5, \mathrm{Ec}=0.01, \mathrm{Rd}=0.5$
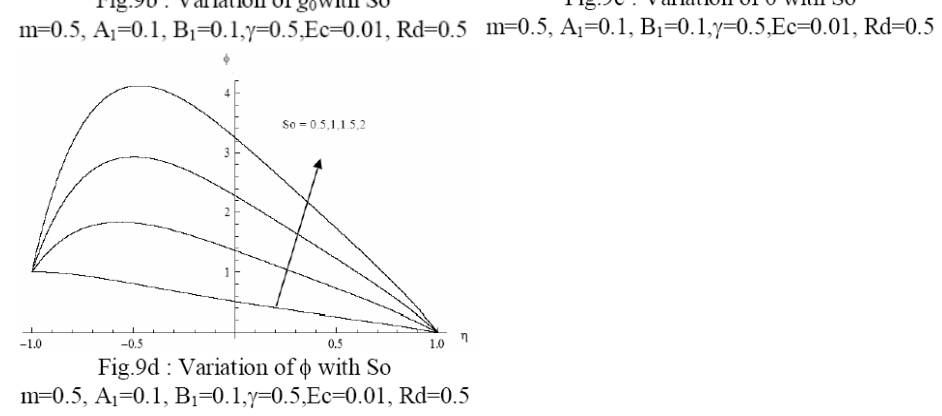

The skin friction components $(\tau \mathrm{x})$ and $(\tau \mathrm{z})$ at the stretching wall $\eta=-1$ and stationary wall $\eta=+1$ are exhibited in tables.2\&3 for different parametric variations. The skin friction with respect to Hall parameter $(m),(\tau x)$ reduces at both the walls while $(\tau z)$ reduces at $\eta=-1$ and enhances at $\eta=+1$. The variation of Stress components with chemical reaction parameter $(\gamma)$ shows that $(\tau x)$ increases at $\eta=-$ 1 and decreases at $\eta=+1$ while $(\tau z)$ reduces both the walls in the degenerating chemical reaction case while, $(\tau x)$ reduces at $\eta=-1$ and enhances at $\eta=+1,(\tau z)$ enhances at both the walls in generating chemical reaction case. Thus lesser the molecular diffusivity larger the skin friction at both the walls. The variation of skin friction components with radiation parameter Rd shows that both the skin friction components reduce at the left wall $\eta=-1$ and enhance at the right wall $\eta=+1$. With reference to heat source parameter $\mathrm{A} 1, \mathrm{~B} 1$ we find that $(\tau \mathrm{x})$ reduces at $\eta=-1$ and enhances at $\eta=+1$ while, $(\tau \mathrm{z})$ enhances at $\eta= \pm 1$ with increase in the space dependent heat generating source $(A 1>0)$ while in the heat absorbing heat source. $(\tau x)$ enhances at at $\eta=-1$ and reduces at $\eta=+1$ while $(\tau z)$ enhances at $\eta=-1$ and reduces at $\eta=+1$.An increase in the temperature dependent heat source $(B 1>0),(\tau x)$ reduces at $\eta=-1$ and enhances at $\eta=+1$ while ( $\tau \mathrm{z})$ enhances at both the walls while in the case of heat absorbing source, $(\tau \mathrm{x})$ and $(\tau \mathrm{z})$ enhances with increase in $\mathrm{B} 1<0$ at both the walls. With increase in Ec, we notice that $(\tau \mathrm{x})$ reduces at $\eta=-1$ and enhances at $\eta=+1$ with increasing in Ec while ( $\tau \mathrm{z})$ increases at both the walls. The variation of Skin friction component with Soret parameter (So) shows that the skin friction component ( $\tau x$ ) reduces at $\eta=-1$ and enhances at $\eta=+1$ while, ( $\tau \mathrm{z})$ increases with So at both the walls. 
Effect of Chemical Reaction, Thermo-Diffusion, Hall Effects on MHD Convective Heat and Mass Transfer Flow of a Viscous Fluid in a Vertical Channel Bounded by Stretching and Stationary Walls

The rate of heat transfer (Nusselt number) at $\eta= \pm 1$ is exhibited in tables. $2 \& 3$ for different parametric variations It is found that the rate of heat transfer enhances at $\eta=-1$ and reduces at $\eta=+1$. An increase in the Hall parameter $(\mathrm{m}),(\mathrm{Nu})$ reduces at both the walls. The rate of heat transfer reduces at $\eta=-1$ and enhances at $\eta=+1$ in the degenerating chemical reaction case and in the generating chemical reaction case, it enhances at $\eta=-1$ and reduces at $\eta=+1$.The rate of heat transfer reduces at $\eta=-1$ and enhances at $\eta=+1$ in the space dependent heat generating source $(A 1>0)$ while in the heat absorbing source, $\mathrm{Nu}$ enhances at $\eta=-1$ and reduces at $\eta=+1$. An increase in B1 $>0$,reduces $\mathrm{Nu}$ at $\eta=-1$ and enhances at $\eta=+1$ while a reversed effect is noticed with increase in $\mathrm{B} 1<0$. The variation of $\mathrm{Nu}$ with radiation parameter $\mathrm{Rd}$ shows that the rate of heat transfer enhances at both the stretching and stationary walls. Higher the dissipation effects smaller $\mathrm{Nu}$ at $\eta=-1$ and larger $\mathrm{Nu}$ at $\eta=+1$.The variation of $\mathrm{Nu}$ with So shows that higher the thermo-diffusion effects smaller $\mathrm{Nu}$ at $\eta=-1$ and larger $\mathrm{Nu}$ at $\eta=+1$.

The rate of mass transfer (Sherwood number) at $\eta= \pm 1$ is exhibited in tables.2\&3 for different parametric variations. An increase in Hall parameter(m) larger the rate of mass transfer at both the stretching and stationary walls. An increase in A1 $>0$ and B1 $>0$, reduces Sh at $\eta=-1$ and enhances at $\eta=+1$ while an increase in $A 1<0$ and $B 1<0$,enhances Sh at the stretching wall and at the stationary wall, it reduces with $\mathrm{A} 1<0$ and enhances with $\mathrm{B} 1<0$.The variation of Sh with radiation parameter $\mathrm{Rd}$

Table2. Skin friction $(\tau)$, Nusselt number $(\mathrm{Nu})$ and Sherwood number(Sh) at $\eta=-1$

\begin{tabular}{|c|c|c|c|c|c|}
\hline \multicolumn{2}{|c|}{ Parameter } & $\tau \times(-1)$ & $\tau z(-1)$ & Nu (-1) & Sh (-1) \\
\hline $\mathbf{m}$ & $\begin{array}{l}0.5 \\
1.0 \\
1.5 \\
2.5 \\
\end{array}$ & $\begin{array}{l}-3.558323 \\
-3.376183 \\
-3.308994 \\
-3.283635 \\
\end{array}$ & $\begin{array}{l}0.221778 \\
0.210876 \\
0.206881 \\
0.159856\end{array}$ & $\begin{array}{l}1.45678 \\
1.39401 \\
1.36009 \\
1.34243\end{array}$ & $\begin{array}{l}-0.093855 \\
-0.139712 \\
-0.157517 \\
-0.164357\end{array}$ \\
\hline Rd & $\begin{array}{l}0.5 \\
1.5 \\
3.5 \\
5.0\end{array}$ & $\begin{array}{l}-3.5583 \\
-3.3981 \\
-3.2547 \\
-3.1730\end{array}$ & $\begin{array}{l}0.221778 \\
0.231004 \\
0.238578 \\
0.477399\end{array}$ & $\begin{array}{l}1.456783 \\
0.736872 \\
0.259932 \\
0.160173\end{array}$ & $\begin{array}{c}-0.093855 \\
1.6763146 \\
2.9624278 \\
3.322490\end{array}$ \\
\hline$\gamma$ & $\begin{array}{r}0.5 \\
1.5 \\
-0.5 \\
-1.5\end{array}$ & $\begin{array}{l}-3.55832 \\
-3.61659 \\
-3.69295 \\
-3.68737\end{array}$ & $\begin{array}{l}0.221778 \\
0.218986 \\
0.188509 \\
0.217923\end{array}$ & $\begin{array}{l}1.45678 \\
1.30545 \\
1.22733 \\
1.28065\end{array}$ & $\begin{array}{l}-0.093855 \\
-0.287123 \\
-0.266086 \\
-0.306148\end{array}$ \\
\hline Ec & $\begin{array}{l}0.01 \\
0.03 \\
0.05 \\
0.07\end{array}$ & $\begin{array}{l}-3.5583 \\
-3.54365 \\
-3.53312 \\
-3.52411\end{array}$ & $\begin{array}{l}0.221778 \\
0.222397 \\
0.222841 \\
0.223224\end{array}$ & $\begin{array}{l}1 . .45678 \\
1.17062 \\
1.06467 \\
0.97424\end{array}$ & $\begin{array}{l}-0.093855 \\
0.5430821 \\
0.9994092 \\
1.3888133\end{array}$ \\
\hline A1 & $\begin{array}{c}-0.1 \\
-0.3 \\
0.1 \\
0.3 \\
\end{array}$ & $\begin{array}{l}-3.558323 \\
-3.554554 \\
-3.566142 \\
-3.574283 \\
\end{array}$ & $\begin{array}{l}0.221778 \\
0.222247 \\
0.221334 \\
0.220868\end{array}$ & $\begin{array}{l}1 . .45678 \\
1.28385 \\
1.35093 \\
1.38484\end{array}$ & $\begin{array}{c}-0.0938553 \\
0.0214518 \\
-0.202995 \\
-0.31689\end{array}$ \\
\hline B1 & $\begin{array}{c}-0.1 \\
-0.3 \\
0.1 \\
0.3 \\
\end{array}$ & $\begin{array}{l}-3.558323 \\
-3.546926 \\
-3.568762 \\
-3.579343 \\
\end{array}$ & $\begin{array}{l}0.221778 \\
0.222432 \\
0.221175 \\
0.220562 \\
\end{array}$ & $\begin{array}{c}1 . .45678 \\
1.27367 \\
1.35969 \\
1.4019\end{array}$ & $\begin{array}{l}-0.0938553 \\
-0.0510332 \\
-0.2289141 \\
-0.3678362 \\
\end{array}$ \\
\hline S0 & $\begin{array}{l}0.5 \\
1.0 \\
1.5 \\
2.0\end{array}$ & $\begin{array}{l}-3.558323 \\
-3.526582 \\
-3.429623 \\
-3.296553\end{array}$ & $\begin{array}{l}0.221778 \\
0.223398 \\
0.228031 \\
0.233727\end{array}$ & $\begin{array}{l}1 . .45678 \\
1.32602 \\
1.34873 \\
1.37823\end{array}$ & $\begin{array}{r}-0.0938553 \\
-1.2882922 \\
-4.9872624 \\
-10.184867\end{array}$ \\
\hline
\end{tabular}

Table3. Skin friction $(\tau)$, Nusselt number $(N u)$ and Sherwood number(Sh) at $\eta=+1$

\begin{tabular}{|c|c|c|c|c|c|}
\hline \multicolumn{2}{|c|}{ Parameter } & $\boldsymbol{\tau x}(+\mathbf{1})$ & $\boldsymbol{\tau} \mathbf{( + 1 )}$ & $\mathbf{N u}(+\mathbf{1})$ & Sh (+1) \\
\hline $\mathbf{m}$ & 0.5 & -0.0391878 & -0.0015796 & 0.0054965 & 0.620306 \\
& 1.0 & -0.0387895 & -0.0018626 & 0.0045104 & 0.622345 \\
& 1.5 & -0.0386047 & -0.0019990 & 0.003545 & 0.623083 \\
& 2.5 & -0.0385294 & -0.0027354 & 0.0022949 & 0.623354 \\
\hline Rd & 0.5 & -0.0391878 & -0.0015796 & 0.005496 & 0.620306 \\
& 1.5 & -0.0552975 & -0.0018187 & 0.283757 & 0.521261 \\
& 3.5 & -0.0713023 & -0.0019719 & 0.496397 & 0.260198 \\
\hline
\end{tabular}


Effect of Chemical Reaction, Thermo-Diffusion, Hall Effects on MHD Convective Heat and Mass Transfer Flow of a Viscous Fluid in a Vertical Channel Bounded by Stretching and Stationary Walls

\begin{tabular}{|c|c|c|c|c|c|}
\hline & 5.0 & -0.1020483 & -0.0019898 & 0.544073 & 0.178378 \\
\hline$\gamma$ & 0.5 & -0.0391878 & -0.0015796 & 0.005496 & 0.620306 \\
& 1.5 & -0.0354346 & -0.0015168 & 0.134073 & 0.267904 \\
& -0.5 & 0.07213942 & -0.0035276 & 0.184716 & -4.74675 \\
& -1.5 & -0.1116515 & -0.0036771 & 0.137416 & -5.55064 \\
\hline Ec & 0.01 & -0.0391878 & -0.0015796 & 0.005496 & 0.620306 \\
& 0.03 & -0.0398127 & -0.0015869 & 0.133794 & 0.626989 \\
& 0.05 & -0.0402662 & -0.0015920 & 0.137199 & 0.631786 \\
& 0.07 & -0.0406572 & -0.0015964 & 0.140174 & 0.635882 \\
\hline A1 & -0.1 & -0.0391878 & -0.0015796 & 0.0054965 & 0.620306 \\
& -0.3 & -0.0398031 & -0.0015895 & 0.133617 & 0.624134 \\
& 0.1 & -0.0386036 & -0.0015699 & 0.124996 & 0.616737 \\
& 0.3 & -0.0379923 & -0.0015594 & 0.120696 & 0.613068 \\
\hline B1 & -0.1 & -0.0391878 & -0.0015796 & 0.005496 & 0.620306 \\
& -0.3 & -0.0400879 & -0.0015945 & 0.135795 & 0.624374 \\
& 0.1 & -0.0383642 & -0.00156546 & 0.123219 & 0.616539 \\
& 0.3 & -0.0375332 & -0.00155076 & 0.117336 & 0.612686 \\
\hline S0 & 0.5 & -0.0391878 & -0.0015796 & 0.135496 & 0.620306 \\
& 1.0 & -0.0420555 & -0.0016288 & 0.126283 & 0.908078 \\
& 1.5 & -0.0500124 & -0.0017414 & 0.118059 & 1.781734 \\
& 2.0 & -0.0592281 & -0.0018263 & 0.108038 & 2.964065 \\
\hline
\end{tabular}

\section{CONCLUSiON}

The nonlinear coupled equations governing the flow heat and mass transfer have solved by using RungeKutta shooting technique. From the profiles and tabular values, we find that;

- An increasing hall parameter, increases the primary velocity, concentration while secondary velocity and temperature reduces in the flow region. The Nusselt number reduces and Sherwood number enhances with increasing $m$.

- Higher the radiation heat flux larger the velocity, component, temperature and smaller the concentration. The Nusslet number enhances the Sherwood number decreases with Rd.

- Higher the thermo-diffusion effect, larger the velocity component and concentration and smaller the temperature. The Nusselt number reduces and Sherwood number enhances with increasing $\mathrm{S}_{0}$.

- An increasing Eckert number $(\mathrm{Ec})$ enhances a velocity component, temperature and reduces the concentration. Also the rate of heat and mass transfer enhances with increase on the wall.

- The primary velocity and the temperature and the secondary velocity, concentration reduce in the degenerating chemical $(\gamma)$ reaction case and in the generating chemical reaction $(\gamma)$ case the velocity components and the concentration enhances and the temperature reduces in the entire flow region

- An increasing space dependent heat source parameter (A1) reduces primary velocity, enhances the secondary velocity, temperature and concentration with A1 $>0$, while reversed effected is noticed with $\mathrm{A} 1<=0$.

- An increasing temperature dependent heat source parameter (B1) enhances a velocity, temperature and reduces concentration with $\mathrm{B} 1>0$, while the experience depreciation with $\mathrm{B} 1<0$.

\section{REFERENCES}

[1] Ashraf,M,Kamal,M.A and Syed,K.S: Numerical study of asymmetric laminar flow of micropolar fluids

[2] in a porous channel .,Computers and fluid., V.38, pp.1895-1902(2009)

[3] Asia,Y,Kashif,A and Muhammad,A:MHD Unsteady flow and heat transfer of micropolar fluid through porous channel with expanding or contracting walls., JAFM, V.9(4),pp.1807-1817(2016)

[4] Berman,A.S:Laminar flow in channels with porous walls.,J.Appl.Phys,V.24, pp.1232-1235(1953)

[5] Bhattacharya,K and Layek,G.C:Slip effect on diffusion of chemically reactive species in boundary layer flow over a vertical stretching sheet with suction or blowing.,Chemical Eng.Commun.,V198,p.1354(2011)

[6] Brady,J.F: Flow development in a porous channel and tube, Phys. Fluids, V.27, pp.1061-1067(1984)

[7] Cox,S.M:Analysis of steady flow in a channel with one porous wall or with accelerating walls, SIAQM J.Appl.Math, V.51,pp.429-438(1991) 
Effect of Chemical Reaction, Thermo-Diffusion, Hall Effects on MHD Convective Heat and Mass Transfer Flow of a Viscous Fluid in a Vertical Channel Bounded by Stretching and Stationary Walls

[8] Crane,I.J:Flow past a stretching plate,Z.Angew.Math Phys(ZAMP),V.31,pp.645-647(1970).

[9] Deng,C and Martinez,D.M"Viscous flow in a channel partially filled with a porous medium and with wall suction,Chemical Eng.Sci,V.60,pp.329-336(2005)

[10] Hajipour,M and Dehkord,A.M:Transient behaviour of fluid flow and heat transfer in vertical channel partially filled with porous medium:Effects of inertial term and viscous dissipation.,Energy Conversion and Management,V.61,pp.1-7(2012)

[11] Hayat,T ,Qasim M Abbas Z and Hendi,A.A:Magnetohydrodynamic flow and mass transfer of a Jeffery fluid over a nonlinear stretching surface.,Z.Naturorsch A,C.64a,p.1111(2010)

[12] Hewitt,R.E,Duck,P.W and AL-Azhari: Extension to three -dimensional flow in a porous channel.,Fluid Dynamics Res., V.33, pp.17-39(2003)

[13] Kandasamy,R,Periasamy K and Prabhu K.K .S:Chemical reaction, heat and mass transfer on MHD flow over a vertical stretching surface with heat sources and thermal stratification effects.,Int.J.Heat Mass Transfer, V.48,p.4751(2005)

[14] Karode,S.K:Laminar flow in channels with porous walls.,Revisited J.Membranes Sci.,V.191,pp.237241(2001)

[15] Kashif Ali,Muhammad Ashraf:Numerical simulation of the micropolar fluid flow and heat transfer in a channel with a shrinking and stationary wall,J.Theo. and Appl mech.,V.52(2),pp.557-569(2014)

[16] Laurent,O,Philippe,S and Michel,Q: Laminar flow in channes with wall suction or injection .,Chemical Eng Sci., V59, pp.1039-1050(2004)

[17] Madhavi,S and Prasada Rao,D.R.V:effect of chemical reaction and thermo-diffusion on convective heat and mass transfer flow of a rotating fluid through a porous medium in a vertical channel with stretching walls.,Int.J.Math. Archives, 8(5), pp.65-79(2017)

[18] Makinde,O.D:MHD mixed convection interaction with thermal radiation and $\mathrm{n}$ th order chemical reaction past a vertical stretching porous plate embedded in a porous medium.,Chem.Eng.Commun.V.198,p.590 (2011)

[19] Mishra J.C,Pal,B., Gupta A.S:Hall effects on magnetichydrodynamic flow and heat transfer over a stretching surface .In: Malik SK, editor.Mathematics and its application to industry and new engineering area, New Delhi:INSA,pp.49-62 (2001)

[20] Mishra,J.C,Shit ,G.C and Rath ,H.J:Flow and heat transfer of a MHD viscoelstic fluid in a channel with stretching walls:Some applications to Haemo-dynamics., ZARM,pp.1-26(2010)

[21] Muhammad Ashraf and Muhammad Abu Bakar:Micropolar fluid flow in achannel with Shrinking walls, World Appl. sci.J.. V. 115, (8), pp.1074-1081(2011)

[22] Pudi Sreenivasa Rao : MHD Mixed Convective Heat and Mass Transfer in a Vertical Channel with Stretching Walls, International Journal for Research \& Development In Technology, V-7(4), pp.21-32,(2017), ISSN (O) :- 2349-3585, www.ijrdt.org.

[23] Qi,X.G,Scott,D.M and Wilson,D.I:Modling laminar pulsed flow in rectangular channel.,Eng. sci., V.63, pp.2682-2689(2008)

[24] Raftari,B and Vajravelu,K:Homotophy analysis method for MHD viscoelaqstic fluid flow and heat transfer in a channel with a stretching wall.,Commun Nonlinear sci Numer Simulat,V.17,pp.4149-4162 (2012)

[25] Rawool,A.S Mitra,S.K and Kandilkar,S.G:Numerical simulation of flow through microchannels with designed roughness., Microfluids and Nanofluids.,V.2, pp.215-221 (2006)

[26] Robinson,W.A:The existence of multiple solutions for the laminar flow in a uniformly porous channel with suction at both walls.,J.Fluid.Mech.,V,212,pp.451-485(1990)

[27] Sarojamma,G,Vasundhara,B and Vendabai, K : MHD Casson fluid flow,heat and mass transfer in a vertical channel with stretching walls., Int.J.Sci and Innovative Mathematical Res,V.2(10), pp.800-810(2014)

[28] Shrestha,G.M and R.M.Terril:Laminar flow through parallel and uniformly porous walls of different permeability, ZAMP, V.16,pp.470-482(1965)

[29] Shrestha,G.M and R.M.Terril:Laminar flow with large injection through parallel and uniformly porous walls of different permeability,Qurt.J.Mech and Appl. Mech., V.21, pp.413-432(1968)

[30] Sukanya,J.S and Leelarathnam, A: Convective heat and mass transfer flow in a rotating vertical channel bounded by stretching and stationary walls with soret effect and thermal radiation, chemical reaction in the presence of non-uniform heat sources, I.J.M.A., Vol.9(9), pp.1-10, (2018)

[31] Sutton,R.S and Barto,A.G:Exact Navier-stokes solution for pulsatory viscous channel flow with arbitrary pressure gradient. J.Propulsiuon and Power,V.24,pp.1412-1424(2008) 
Effect of Chemical Reaction, Thermo-Diffusion, Hall Effects on MHD Convective Heat and Mass Transfer Flow of a Viscous Fluid in a Vertical Channel Bounded by Stretching and Stationary Walls

[32] Taylor,C.L, Banks, W.H.H , Zaturska,M.B and Drazin,P.G:Three dimensional flow in porous channel., Quart. J.Mech.Appl., V.44, pp.105-133(1991)

[33] Terrill,R.M:Laminar flow in a uniformly porous channel with large injection., Aeronaut. Q.,V.15,pp. 299310(1965)

[34] Wang,C.Y:Analysis of viscous flow due to a stretching sheet with surface slip and suction,J.Nonlinear Analysis.Real World Applications,V.10,pp.375-380(2009)

[35] Watson,E.B and Banks,W.H.H, Zaturska, M.B and Drazen,F.G:On transition to chaos in two dimensional channel flow symmetrically driven by accelerating walls., J.Fluid Mech.,V.212,pp.451-485 (1990)

[36] Xinhui Si , Liancum Zheng,Ping Lin, Xinxin zhang,Yan Zhang:Flow and heat transfer of a micropolar fluid in a porous channel with expandind or Contracting wall,Int.J.Heat and Mass transfer, V.67, pp.885-895(2013)

[37]Zheng,L,Zhao,N and Zhang,X: Asymptotic solutions for laminar flow in a channel with uniformly accelerating rigid porous walls., J. University of Sci and technology Beijeing,V.14.pp405-409(2007)

\section{AUTHORS' BIOGRAPHY}

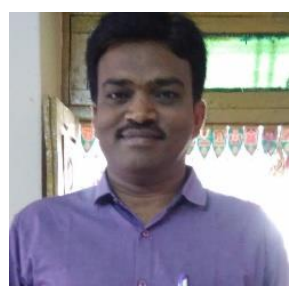

K.Chandrasekhar Raju, has M.Sc. graduated from Sri Venkateswara University, Tirupati, Andhra Pradesh, India in 2002. He is currently a Ph.D. Research Scholar in Dravidian University, Kuppam, A.P., India. Presently working as a Lecturer, S.V.Degree College, Kadapa, A.P., India, since 2003. His current area of research are in field of Fluid Dynamics, Heat and Mass transfer, Micro Polar Fluid.

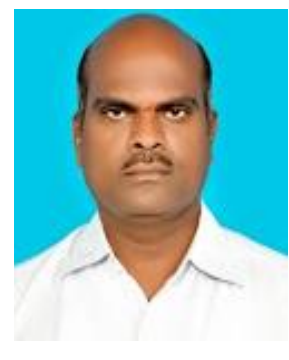

Dr. B. Sreenivasa Reddy, is an Assistant Professor, Department of Applied Mathematics, Yogivemana University, Kadapa, A.P., India. He did his M.Sc., M.Phil., from Sri Krishnadevaraya University Anantapur, India in 1997,2002 and earned his Ph.D. from the same University in 2006. He has over 13 years of teaching experience and groomed many graduate and postgraduate students and researcher scholars. His areas of interest are Fluid Dynamics, bio-mechanics, Heat and mass Transfer in Porous Medium, Chemical Reaction in Fluid Flows and Nano fluids. He has published 30 papers in archival journals and conferences.

Citation: Dr. B. Sreenivasa Reddy \& K.Chandrasekhar Raju, (2019). Effect of Chemical Reaction, ThermoDiffusion, Hall Effects on MHD Convective Heat and Mass Transfer Flow of a Viscous Fluid in a Vertical Channel Bounded by Stretching and Stationary Walls. International Journal of Scientific and Innovative Mathematical Research (IJSIMR), 7(4), pp.12-24. http://dx.doi.org/10.20431/2347-3142.0704003

Copyright: (C) 2019 Authors, this is an open-access article distributed under the terms of the Creative Commons Attribution License, which permits unrestricted use, distribution, and reproduction in any medium, provided the original author and source are credited. 